\title{
Treatment with imatinib improves drug delivery and efficacy in NSCLC xenografts
}

\author{
G Vlahovic ${ }^{*, 1,6}$, AM Ponce ${ }^{2,3,6}$, Z Rabbani ${ }^{4}$, FK Salahuddin ${ }^{5}$, L Zgonjanin ${ }^{4}$, I Spasojevic', Z Vujaskovic ${ }^{4}$ and \\ MW Dewhirst ${ }^{2,4}$
}

'Department of Medicine - Oncology, Duke University Medical Center, PO Box 3335, Durham, NC 277I0, USA; ${ }^{2}$ Department of Biomedical Engineering, Duke University, Durham, NC, USA; ${ }^{3}$ School of Medicine, Duke University, Durham, NC, USA; ${ }^{4}$ Department of Radiation Oncology, Duke University Medical Center, Durham, NC, USA; ${ }^{5}$ Department of Medicine, Durham Regional Hospital/Duke University Medical Center, Durham, NC, USA

Imatinib, an inhibitor of PDGF-R $\beta$ and other tyrosine kinase receptors, has been shown to decrease microvessel density and interstitial fluid pressure in solid tumours, thereby improving subsequent delivery of small molecules. The purpose of this study was to test whether pretreatment with imatinib increases the efficacy of traditional chemotherapy in mice bearing non-small cell lung carcinoma xenografts, and to investigate the effects of imatinib on liposomal drug delivery. Efficacy treatment groups included ( $n=9-$ 10): saline control, imatinib alone (oral gavage, $100 \mathrm{mg} \mathrm{kg}^{-1} \times 7$ days), docetaxel alone $\left(10 \mathrm{mg} \mathrm{kg}^{-1}\right.$ i.p. $2 \times /$ week until killing), and imatinib plus docetaxel (started on day 7 of imatinib). Tumours were monitored until they reached four times the initial treatment volume $(4 \times V)$ or 28 days. A separate experiment compared tumour doxorubicin concentrations (using high performance liquid chromatography) $24 \mathrm{~h}$ after treatment with liposomal doxorubicin alone $\left(6 \mathrm{mg} \mathrm{kg}^{-1}\right.$ i.v., $\left.n=9\right)$ or imatinib plus liposomal doxorubicin $(n=16)$. Imatinib plus docetaxel resulted in significantly improved antitumour efficacy $(0 / 10$ animals reached $4 \times V$ by 28 days $)$ when compared to docetaxel alone $(3 / 9$ reached $4 \times V, P=0.014)$ or imatinib alone $(9 / 10$ reached $4 \times V, P=0.025)$. Pretreatment with imatinib also significantly increased tumour concentrations of liposomal doxorubicin. Overall, these preclinical studies emphasise the potential of imatinib as an adjunct to small molecule or liposomal chemotherapy.

British Journal of Cancer (2007) 97, 735-740. doi:I0.1038/sj.bjc.660394I www.bjcancer.com

Published online 21 August 2007

(c) 2007 Cancer Research UK

Keywords: lung cancer; imatinib; drug delivery; interstitial fluid pressure

Non-small cell lung carcinoma (NSCLC) has a limited response rate to current chemotherapeutics, with tumour shrinkage in only $20 \%$ of patients (by the RECIST criteria) and 2-year survival rates of 10-16\% (Schiller et al, 2002). One of the major barriers to effective chemotherapy is the lack of adequate drug delivery to lung tumours, which has been linked to high tumour interstitial fluid pressures (IFPs) of up to $25 \mathrm{~mm} \mathrm{Hg}$ (Jain, 1996). This challenge has also been demonstrated for many other solid tumours, including breast carcinoma, head and neck carcinoma, and melanoma (Jain, 1996; Heldin et al, 2004). Loss of pressure gradient across the microvessel wall inhibits drug transport from blood to the interstitium. In fact, elevated pressure throughout the core of solid tumours often results in drug distribution only to the periphery of tumours after intravenous injection (Boucher et al, 1990; Viglianti et al, 2004). Therefore, the rationale is quite strong for developing a method to improve tumour drug delivery by reducing IFP (Heldin et al, 2004; Jain, 2005).

Systemic chemotherapeutics encounter a number of obstacles that limit transport to solid tumours (Jain, 2001; Truskey et al, 2004). First, tumour angiogenesis results in immature vasculature with abnormal architecture and leaky, heterogeneous vessel walls (Yuan et al, 1995; Yuan, 1998). Vessels may also be compressed by

\footnotetext{
*Correspondence: Dr G Vlahovic; E-mail: vlaho00I@mc.duke.edu

${ }^{6}$ These authors contributed equally to this work.

Revised 23 July 2007; accepted 25 July 2007; published online 21 August 2007
}

the solid stress of proliferating tumour parenchyma and stroma (Boucher and Jain, 1992; Griffon-Etienne et al, 1999). These factors are known to impair vascular perfusion, thereby limiting drug access to the tumour. As noted above, another major problem is interstitial hypertension, which decreases convective transport of drug across the microvessel wall (Boucher et al, 1990; Heldin et al, 2004). Elevated IFP has been attributed to leakage of plasma proteins and fluid from permeable tumour vessels with high intravascular pressure. Tumours lack functional lymphatics to remove the excess interstitial fluid, and pressure equilibrates across the wall (Boucher and Jain, 1992). Furthermore, fibroblasts and pericytes have been shown to contribute to elevated IFP by exerting PDGF-mediated tension on the interstitial collagen matrix (Heldin et al, 2004). The PDGF receptors, located on stroma and perivascular cells, dimerise and bind PI3K, leading to integrinmediated action on collagen fibers (Heuchel et al, 1999; Pietras et al, 2001).

Non-small cell lung carcinoma exhibits high IFP, upregulated PDGF, and a poor clinical prognosis, making this a particularly valuable model for impaired drug delivery (Kawai et al, 1997; Vlahovic et al, 2006). Interstitial hypertension particularly affects macromolecular therapies such as liposomes, antibodies, and proteins, as they require convective transport to cross the capillary wall (Jain and Baxter, 1988; Yuan, 1998; Netti et al, 1999). The current strategies for reducing IFP include blockage of cytokines (e.g. VEGF, PDGF, and TGF- $\beta$ ) and decompression of vessels by induction of tumour cell death (Boucher and Jain, 1992; 
Griffon-Etienne et al, 1999). Blocking VEGF prunes immature vessels, increases fraction of pericyte-covered vessels, and decreases vessel permeability, dilation and tortuosity (Tong et al, 2004; Willett et al, 2004; Jain, 2005). Inhibition of PDGF or PDGF$\mathrm{R} \beta$ also prunes immature vessels, and additionally decreases contraction of the interstitial space and proliferation of stroma (Heuchel et al, 1999; Pietras et al, 2001; George, 2003; Matei et al, 2004; Vlahovic et al, 2006).

Imatinib (Gleevec, Novartis Pharmaceuticals Corp., East Hanover, NJ, USA) is a synthetic small molecule that inhibits phosphorylation of a number of tyrosine kinase receptors including PDGFR, c-kit, and bcr-abl (Buchdunger et al, 1995; George, 2003; Matei et al, 2004). It decreases proliferation of both tumour and stromal cells (George, 2003; Furuhashi et al, 2004; Kim et al, 2004; Matei et al, 2004; Ostman, 2004). In colonic xenografts, Pietras et al (2001) demonstrated that imatinib decreases IFP and thereby increases transcapillary transport of small molecules. We have recently reported the effects of imatinib on NSCLC xenografts, showing that 4 days of therapy $\left(50 \mathrm{mg} \mathrm{kg}^{-1}\right.$ gavage) significantly decreases IFP and microvascular density, lowers VEGF levels, and improves tumour oxygen delivery (Vlahovic et al, 2006). In the current study, we have continued this work by investigating whether pretreatment with imatinib can be used to affect tumour vasculature and consequently improve the efficacy of traditional chemotherapeutics in NSCLC xenografts. Furthermore, we provide a proof of principle for the use of imatinib to improve liposomal drug delivery in this model.

\section{MATERIALS AND METHODS}

\section{Materials}

Imatinib (Gleevec, STI571, Novartis Pharmaceuticals Corp.) was obtained from the hospital pharmacy in a 100-mg tablet form. Tablets were crushed and re-suspended in $0.9 \% \mathrm{NaCl}$. Liposomal doxorubicin (Doxil) was obtained from Ortho Biotech Products (Bridgewater, NJ, USA), and docetaxel (Taxotere) was obtained from Sanofi Aventis (Bridgewater). A human NSCLC line, A549, was purchased from American Type Culture Collection (Manassas, VA, USA).

\section{Non-small cell lung carcinoma xenograft model}

Homozygous adult female athymic nude mice were housed in isolated caging with sterile rodent food and water ad libitum. Human NSCLC cells (A549) were grown in RPMI 1640 media supplemented with $5 \%$ bovine calf serum. The right hind limb of each mouse was inoculated subcutaneously with $\sim 5.0 \times 10^{5}$ A549 cells, obtained from exponentially growing cell cultures, suspended in $200 \mu$ l of HEPES-buffered saline. Xenograft tumour growth was monitored twice per week until tumours reached an average volume of $100 \mathrm{~mm}^{3}$. Xenograft volume $(V)$ was determined by the following equation: $V=\left(L \times W^{2}\right) \times 0.5$, where $L$ is the length and $W$ is the width of the flank tumour.

\section{Therapy and tumour harvest}

After xenografts reached treatment volume $\left(100 \mathrm{~mm}^{3}\right)$, tumourbearing animals were used in three separate studies. The first study compared vascular maturity (by immunohistochemistry) after treatment with imatinib $(n=4) v s$ control (saline, $n=5)$. Imatinib was administered via gavage once a day at a dose of $100 \mathrm{mg} \mathrm{kg}^{-1}$ for 7 days, and saline was administered following the same schedule. Animals were killed $24 \mathrm{~h}$ after the last imatinib injection, and tumours were harvested and snap frozen at $-80^{\circ} \mathrm{C}$.

The second study compared tumour growth times after treatment with control (saline plus saline), imatinib alone (plus saline), docetaxel alone (plus saline), or imatinib plus docetaxel
( $n=9-10$ mice per group). Docetaxel was administered intraperitoneally at a dose of $10 \mathrm{mg} \mathrm{kg}^{-1}$ twice per week until killing, and saline was administered following the same schedule. Injections of docetaxel (docetaxel \pm imatinib groups) or intraperitoneal saline (control + imatinib alone groups) were initiated on day 7 of imatinib or saline pretreatment. After therapy, animals were monitored as they recovered from anaesthesia. Tumour volumes were measured three times per week for 28 days or until the tumour volume reached four times the volume on the first day of treatment.

The third study compared tumour doxorubicin concentrations after treatment with liposomal doxorubicin alone $(n=9)$ vs imatinib plus liposomal doxorubicin $(n=16$, randomised in $1: 2$ ratio). Liposomal doxorubicin was delivered intravenously as a single dose $\left(6 \mathrm{mg} \mathrm{kg}^{-1}\right)$. When combined with imatinib, liposomal doxorubicin was delivered on day 7 of imatinib treatment. Animals were killed 1 day $(24 \mathrm{~h})$ after liposomal doxorubicin injection, and tumours were harvested and snap frozen at $-80^{\circ} \mathrm{C}$. All animal protocols were approved by the Duke University Animal Care and Use Committee. Experiments were performed in accordance with the Interdisciplinary Principles and Guidelines for the Use of Animals in Research, Marketing, and Education (New York Academy of Sciences, New York, NY, USA), as well as the UKCCCR guidelines (Anon, 1988).

\section{Immunohistochemical staining}

Vascular markers for this study included the endothelial cell markers CD31 and CD105, as well as smooth muscle alpha-actin $(\alpha$-SMA). CD31 was used to stain all endothelial cells, whereas CD105 was used to indicate immature endothelial cells. Smooth muscle $\alpha$-actin was used to stain pericytes covering microvessels. Frozen tumours harvested from control and imatinib alone groups were cut into $10-12 \mu \mathrm{m}$ sections using a LEICA CM 1850 cryotome (Meyer Instruments Inc., Houston, TX, USA). Consecutive tissue sections were cut at the largest circumference of the tumour and placed onto poly-L-lysine-coated slides (Polysciences Inc., Warrington, PA, USA). Cryosections were then split into two sets for staining. All antibodies were diluted in phosphate-buffered saline (PBS) with $0.1 \%$ bovine serum albumin and $0.1 \%$ Tween-20 (PBS-BT).

In the first set, sections were stained for CD31 and $\alpha$-SMA. Cryosections were air dried and fixed for $10 \mathrm{~min}$ in acetone at $4{ }^{\circ} \mathrm{C}$. Tumour sections were then blocked with $10 \%$ normal donkey serum for $15 \mathrm{~min}$ and rinsed three times for $2 \mathrm{~min}$ in PBS. Sections were probed sequentially with primary antibodies against CD-31 (rat anti-mouse, diluted 1:100, BD Pharmingen, BD Biosciences, San Jose, CA, USA) and $\alpha$-SMA (mouse monoclonal antibody, clone IA4, diluted 1:400, Lab Vision Corp., Fremont, CA, USA). After rinsing again with PBS, primary antibodies were respectively revealed with secondary antibodies coupled to TRITC (red, for CD-31) or FITC (green, for $\alpha$-SMA) (Jackson Immunoresearch, West Grove, PA, USA).

In the second set, sections were stained for CD31 and CD105. Sections were fixed for $1 \mathrm{~h}$ in $4 \%$ paraformaldehyde in PBS $(\mathrm{pH}$ 7.2). Endogenous peroxidase activity was quenched with $3 \%$ hydrogen peroxide for $15 \mathrm{~min}$, and tumour sections were then blocked with $10 \%$ normal donkey serum for $15 \mathrm{~min}$ and rinsed three times for $2 \mathrm{~min}$ in PBS. Consecutive sections were incubated overnight at $4{ }^{\circ} \mathrm{C}$ with rat monoclonal antibody to CD31 diluted 1:200 or CD105 diluted 1:400 (BD Biosciences, San Jose, CA, USA). After rinsing again with PBS, primary antibodies were enhanced with biotinylated donkey anti-rat secondary antibody (diluted $1: 100$ ) for $30 \mathrm{~min}$ at room temperature (Jackson Immunoresearch). Finally, an avidin-biotin complex was applied (Vectastain ABC kit, Vector Lab Inc., Burlingame, CA, USA). Markers were visualised with $3,3^{\prime}$-diaminobenzidine tetrahydrochloride (DAB chromogen, Vector Lab Inc.). 


\section{Image analysis}

Tumour sections were quantitatively analysed using a semiautomatic computerised digital image analysis system (Metamorph Imaging System, Molecular Devices Corp, Sunnyvale CA, USA). A high-resolution intensified solid-state camera was mounted on a fluorescence microscope (Axioskop, Zeiss, Axioskop Zplus, Carl Zeiss Inc., Germany) with a computer-controlled motorised stepping stage. For the first set (CD31 vs $\alpha$-SMA), digitised images were acquired with both TRITC and FITC filters, and images were overlaid using the Metamorph software. Microvessel density was determined by counting CD31-positive structures in five random fields per tumour, and $\alpha$-SMA-positive vessels were counted from among these structures.

For the second set (CD31 vs CD105), quantification of vessels was performed using the Image J 'Analyze Particles' function by a blind observer without any manipulation in brightness or contrast. The threshold intensity was manually set above the background staining intensity, and the Image J function selected for stained vessels with a minimum size of 10 pixels per vessel. The average number of CD31-stained vessels per region of interest (over three regions per animal) was calculated as the microvessel density. The average number of CD105-stained vessels in the same regions was calculated from the consecutive section.

\section{High performance liquid chromatography for doxorubicin concentration}

Tumours harvested from the liposomal doxorubicin group and the imatinib plus liposomal doxorubicin group were cryo-crushed at $-80^{\circ} \mathrm{C}$, thawed, diluted, and homogenised. Three samples were analysed from each tumour. Doxorubicin was extracted from the homogenate using chloroform and silver nitrate. The organic phase was separated, dried, and reconstituted in isopropanol. Doxorubicin concentration was then measured by high performance liquid chromatography (HPLC) with fluorimetry (Cummings, 1985; Kong et al, 2000). A standard concentration set was prepared using tumour homogenate and known concentrations of doxorubicin. To correct error in weight measurement due to variable tissue water content, concentrations were normalised by total water-soluble protein concentration, which was measured by the Bradford assay (colorimetric response of Coomassie Blue G dye).

\section{Statistical methods}

Descriptive summary statistics are presented as mean \pm s.e. of the mean, except for the time-to-four-times-tumour-volume data, which are expressed as median values with $95 \%$ confidence intervals. Statistical analyses of doxorubicin concentration and histologic data were performed using the two-sample Student's $t$-test. For the antitumour efficacy data, the primary end point (time to reach four times the original tumour volume) was analysed using the Cox proportional hazards model to compare groups. Censoring was taken into account for animals that did not reach four times the original treatment volume by the time they were killed (at 28 days). $P$-values less than 0.05 were considered to be statistically significant.

\section{RESULTS}

Since imatinib is known to inhibit PDGF-R $\beta$ and decrease IFP in solid tumours, it has the potential to 'normalise' the tumour microenvironment and thereby improve delivery and efficacy of chemotherapeutic agents. With this rationale, we performed three in vivo translational studies on the effects of imatinib in NSCLC xenografts. We first used histology to test the vascular effects of imatinib, specifically assessing microvessel density (CD31 positivity), pericyte coverage ( $\alpha$-SMA positivity), and endothelial cell immaturity (CD105 positivity). Then, we followed up on recently published data that demonstrate that pretreatment with imatinib increases delivery of small molecules to tumours (Pietras et al, 2001; Vlahovic et al, 2006). We evaluated the antitumour efficacy of imatinib plus docetaxel (a small molecule chemotherapeutic commonly used for NSCLC treatment) in comparison with either agent alone. Finally, as elevated IFP is known to be a major barrier to convective transport of liposomes, we tested whether pretreatment with imatinib can be used to increase tumour delivery of liposomal doxorubicin. These three studies were designed to complement previously published data and increase the understanding of PDGFR $\beta$-mediated effects of imatinib in NSCLC.

Representative images of NSCLC microvessels stained for CD31 (red) and $\alpha$-SMA (green) are shown in Figure 1 after treatment with saline (control) or imatinib. Pericytes (green) can be seen overlying the endothelial cells (red), especially in larger vessels. After a 7-day course of imatinib treatment, the total number of vessels (anti-CD31-stained) decreased significantly from $51.0 \pm 4.7$ to $29.5 \pm 3.2$ (s.e., $P=0.01$, Figure $2 \mathrm{~A}$ ). The number of anti- $\alpha$-SMAstained microvessels, representing mature pericyte-covered vessels, also decreased significantly, from $14.1 \pm 1.2$ to $7.1 \pm 1.3$ $(P=0.02)$. The percentage of total vessels that were positive for $\alpha$ SMA lowered from 28 to $23 \%$ (Figure 2A), indicating a trend towards decreased pericyte coverage after treatment with imatinib (not significantly different).

In a separate set of tumour sections, the average numbers of CD31-stained and CD105-stained vessels were compared in consecutive sections (Figure $2 \mathrm{~B}$ ). Owing to different image analysis
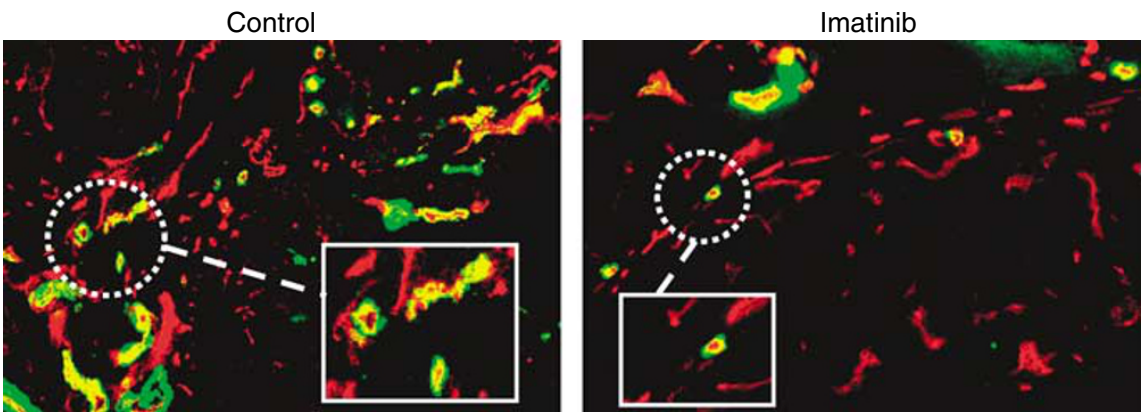

Red $=$ CD31

Green $=\alpha-S M A$

Figure I Representative fluorescent images of NSCLC xenograft sections after treatment with saline control or imatinib, $100 \mathrm{mg} \mathrm{kg}{ }^{-1} \times 7$ days. Images show endothelial cells stained with CD3I (red) and pericytes stained with anti- $\alpha$-SMA (green) 

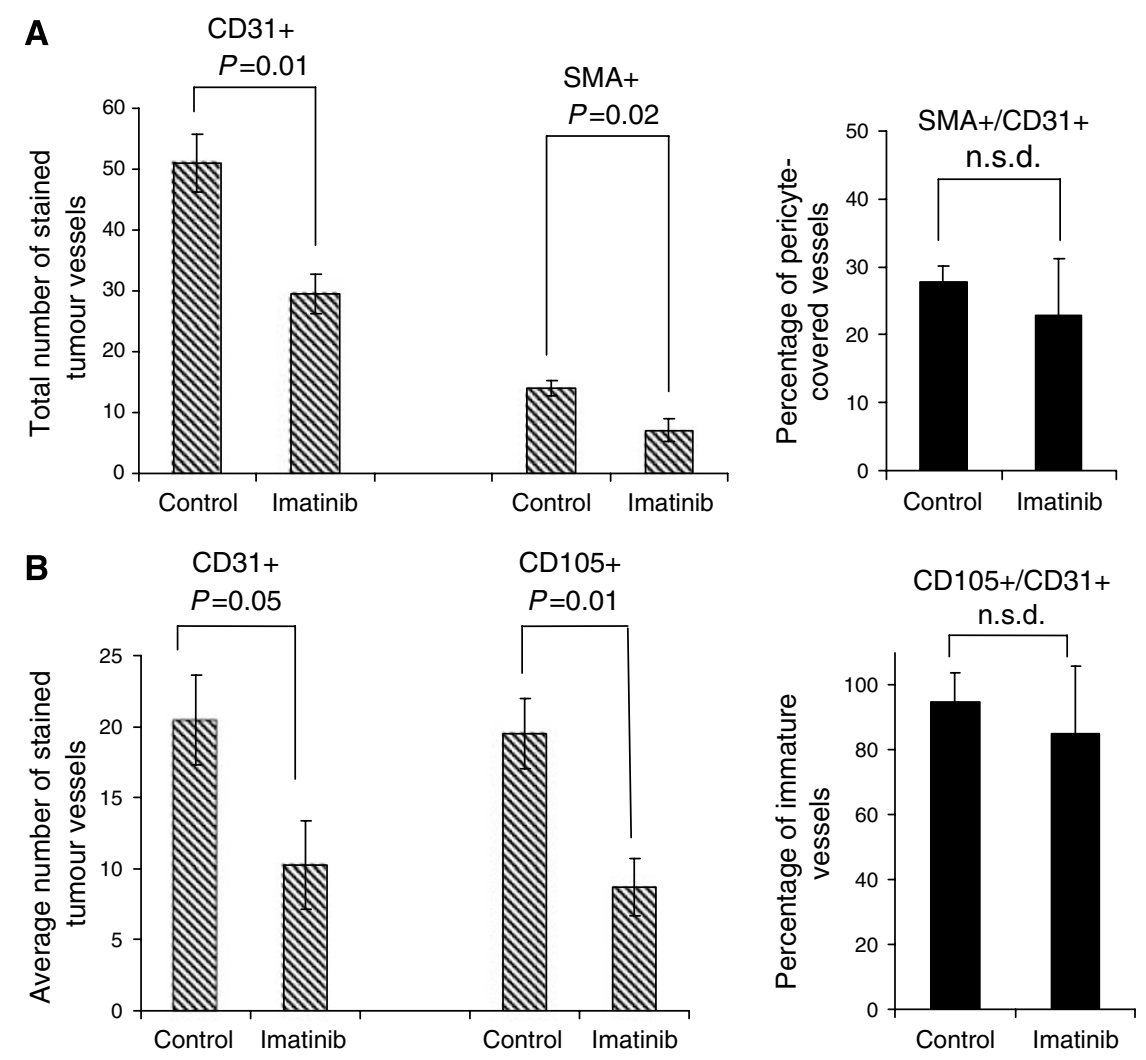

Figure 2 Vascular markers in NSCLC xenografts after treatment with saline control or imatinib, $100 \mathrm{mg} \mathrm{kg}^{-1} \times 7$ days. (A) The total numbers of antiCD31- and anti- $\alpha$-SMA-stained vessels were measured in five regions of the same tumour section. The number of anti-CD 31 -stained vessels represents total microvessel density, and the number of anti- $\alpha$-SMA-stained vessels indicates the extent of pericyte coverage. Data represent mean \pm s.e. (B) The average numbers of anti-CD3I- and anti-CDI05-stained vessels were measured in three regions of consecutive tumour sections. The number of antiCDI05-stained vessels indicates relative endothelial cell immaturity. The number of anti-CD3l-stained vessels differs from $(\mathbf{A})$ due to distinct methods.

methods, the absolute number of CD31-stained vessels in this experiment (Figure 2B) was lower than in the former experiment (Figure 2A), but treatment with imatinib again resulted in a significant decrease in vessel density $(P=0.05)$. The density of immature CD105-stained endothelial cells in the tumour decreased from $19.5 \pm 2.5$ to $8.7 \pm 2.0$ after treatment with imatinib $(P=0.01)$, and the ratio of CD105- to CD31-stained (total) vessels was used as an indicator of vascular immaturity. This ratio was $0.95 \pm 0.09$ in the control tumour and $0.85 \pm 0.21$ after imatinib therapy (Figure $2 \mathrm{~B}$ ), showing a trend towards decreasing vessel immaturity (or increasing maturity).

Figure 3 clearly shows that the combination of imatinib plus docetaxel was significantly more effective than either agent alone in the NSCLC xenograft. When docetaxel was administered after 7 days of imatinib treatment, tumour growth was delayed such that no animals reached the end point of four times the original tumour volume $(4 \times V)$ by 28 days after therapy. In comparison, three out of nine animals receiving docetaxel alone reached $4 \times V$ by 28 days, showing a significantly higher likelihood ratio for reaching $4 \times V(P=0.014$ vs combination). Almost all ( 9 out of 10$)$ animals receiving imatinib alone reached this end point, with a median tumour growth time of $22.5 \pm 3.0$ days (95\% CI). Again, imatinib alone showed a significantly higher likelihood ratio for reaching $4 \times V(P=0.025$ vs combination $)$.

The assessment of imatinib plus liposomal doxorubicin showed that prior treatment with imatinib improves delivery of liposomal doxorubicin to NSCLC tumours (Figure 4). A single dose of $6 \mathrm{mg} \mathrm{kg}^{-1}$ liposomal doxorubicin (without imatinib) resulted in a mean tumour doxorubicin concentration of $530.4 \pm 53.5 \mathrm{ng}$ doxorubicin per mg protein at $24 \mathrm{~h}$ after injection $(n=9)$. Pretreatment with 7 days of $100 \mathrm{mg} \mathrm{kg}^{-1}$ imatinib gavage significantly increased

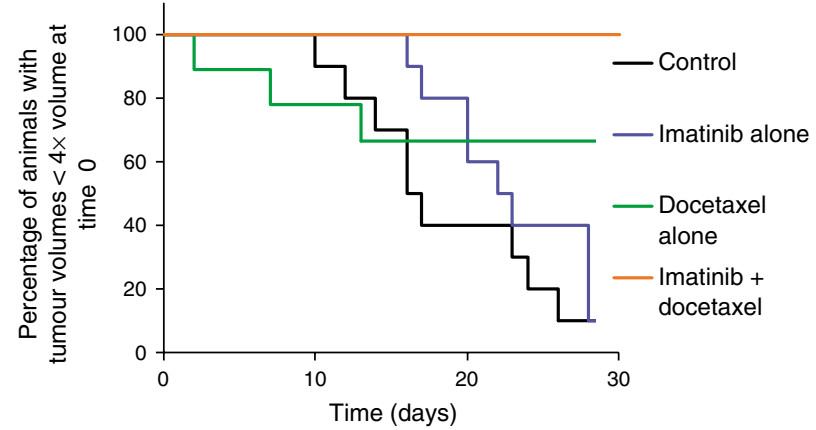

Figure 3 Antitumour response in NSCLC xenografts after treatment with saline, imatinib alone, docetaxel alone, or imatinib plus docetaxel ( $n=9-10$ mice per group). Imatinib dose was $100 \mathrm{mg} \mathrm{kg}^{-1}$ oral gavage $\times 7$ days. Docetaxel dose was $10 \mathrm{mg} \mathrm{kg}^{-1}$ i.p. twice per week, starting on day 7 of imatinib or saline treatment. Tumours were monitored three times per week until they reached four times the initial treatment volume $(4 \times \mathrm{V})$ or 28 days. The Kaplan-Meier plot represents the percentage of animals with tumour volumes less than four times the volume at the beginning of treatment, as a function of time after treatment

the tumour doxorubicin concentration to $779.2 \pm 68.8 \mathrm{ng}$ doxorubicin per mg protein $(n=16, P=0.02)$.

\section{DISCUSSION}

Imatinib has been implicated as a possible effector of vascular 'normalisation', the therapeutic paradigm proposed by Jain (2005) 


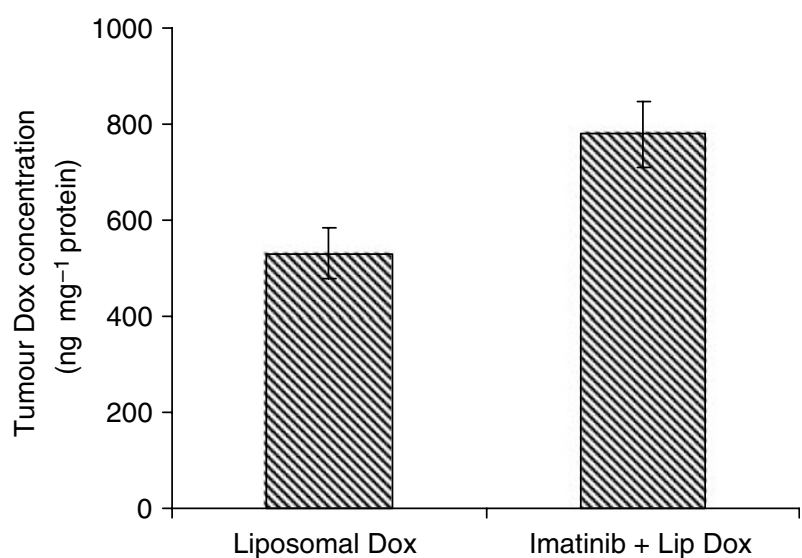

Figure 4 Tumour doxorubicin concentrations (ng doxorubicin per mg protein) $24 \mathrm{~h}$ after therapy with liposomal doxorubicin alone or imatinib plus liposomal doxorubicin. Liposomal doxorubicin was administered i.v. at a dose of $6 \mathrm{mg} \mathrm{kg}^{-1}$. For combined therapy, liposomes were administered on day 7 of imatinib treatment $\left(100 \mathrm{mg} \mathrm{kg}^{-1}\right.$ oral gavage $\times 7$ days). Tumour doxorubicin concentration was measured by HPLC, and data represent mean \pm s.e.

to improve drug and oxygen delivery to tumours. Indeed, in preclinical studies, imatinib therapy has normalised the tumour microenvironment by decreasing IFP, increasing transcapillary transport of oxygen and ${ }^{51} \mathrm{Cr}$-EDTA, and lowering VEGF and microvessel density (Pietras et al, 2001; Vlahovic et al, 2006). The current study further demonstrates a significant decrease in density of immature endothelial cells $(\mathrm{CD} 105+)$, with a trend towards higher endothelial cell maturity (lower CD105-CD31 ratio, Figure $2 \mathrm{~B}$ ). In addition, Figure $2 \mathrm{~A}$ supports a trend towards decreased fraction of pericyte coverage after imatinib therapy. Although not a 'normalising' effect, this result is consistent with imatinib inhibiting the actions of PDGF and PDGF-R $\beta$ in pericyte recruitment and vessel wall stabilisation (George and Kaelin, 2003; Ostman, 2004). Importantly, dosing and scheduling may be crucial if 'normalisation' is the goal (Winkler et al, 2004; Jain, 2005). Future studies should determine the temporal windows of IFP reduction and vascular remodelling induced by imatinib in order to optimise combined therapy schedules (Jain, 2005).

This is the first study to demonstrate the in vivo efficacy of imatinib, as an adjunct to chemotherapy in NSCLC treatment. For NSCLC, the most commonly used first line chemotherapeutics include platinum, taxanes, gemcitabine, and vinorelbine (Schiller et al, 2002). The taxane docetaxel was chosen for this preclinical study, because it is FDA approved as a second line single-agent therapy, and it is under clinical investigation as a first-line singleagent therapy. Here we show that imatinib plus docetaxel resulted in significantly improved antitumour efficacy when compared to docetaxel alone or imatinib alone. These results, along with previously published studies (Pietras et al, 2001; Vlahovic et al, 2006), suggest that imatinib improves small molecule drug delivery, which is typically thought to be governed by diffusion
(Heldin et al, 2004). This may be linked to an increase in diffusion by an unknown mechanism, possibly related to vessel remodelling or available volume fraction in the extracellular matrix (Heuchel et al, 1999; Ostman, 2004). However, some evidence suggests that the effects of convection on small molecules may be underestimated, especially in the tumour microenvironment (Pietras et al, 2001; Heldin et al, 2004; Ostman, 2004). Further preclinical studies should investigate the drug delivery effects of this type of combination therapy, and expand application to other tumour models and other chemotherapeutic agents.

This study also provides a proof of principle for the enhancement of liposomal (i.e. macromolecular) drug delivery by reduction of IFP (Yuan et al, 1994, 1995). Macromolecular transport is dominated by convection, which is governed by hydrostatic and oncotic pressures (Starling forces) (Truskey et al, 2004). A decrease in interstitial hydrostatic pressure is expected to increase the transport of macromolecules across the vessel wall (Heldin et al, 2004). Thus, the observed increase in liposomal doxorubicin delivery to NSCLC after pretreatment with imatinib can be explained by the decrease in IFP previously reported by this group in NSCLC (Figure 4, Pietras et al, 2001; Vlahovic et al, 2006).

Overall, the observed effects of imatinib in NSCLC are consistent with the inhibition of PDGFR- $\beta$; these changes in the microenvironment, including decreased IFP and modified vasculature, result in improved drug delivery and efficacy in the preclinical setting. Currently, this hypothesis is being further tested in clinical trials in which patients with metastatic NSCLC are treated with standard cisplatin and docetaxel plus concurrent imatinib. All enrolled patients must be PDGFR- $\beta$ positive by immunohistochemistry, and treatment response is monitored by the RECIST criteria. Patients also participate in a lead-in portion of the study in which dynamic contrast-enhanced (DCE) MRI is performed before and after a 7-day course of imatinib alone, to explore DCE-MRI as a biomarker for changes in the tumour microenvironment. Preliminary data have revealed that DCE-MRI is feasible and demonstrates a decrease in tumour leakage space (interstitial or extracellular extravascular space) after treatment with imatinib. This suggests a decrease in IFP that may imply improvement in tumour drug delivery. However, a major challenge to clinical research in drug delivery is the lack of non-invasive drug imaging methods (Viglianti et al, 2004; Ponce et al, 2007). The phase I portion of the clinical trial is completed, and the phase II portion evaluating the efficacy of combined imatinib plus chemotherapy is currently successfully enrolling. In conclusion, our preclinical studies with imatinib in NSCLC demonstrate its potential to improve tumour drug delivery and efficacy, and this strategy was subsequently rapidly translated into clinical trials.

\section{ACKNOWLEDGEMENTS}

This work was supported by grants from the National Institutes of Health (NIH/NCRR 5K12RR017630-03 and R01 CA40355). We thank Brad Thrasher for assistance with images and James Herndon for statistical analysis of tumour growth data.

\section{REFERENCES}

Anon (1988) UKCCCR guidelines for the welfare of animals in experimental neoplasia. Br J Cancer 58: 109-113

Boucher Y, Jain RK (1992) Microvascular pressure is the principal driving force for interstitial hypertension in solid tumors: implications for vascular collapse. Cancer Res 52: 5110-5114

Boucher Y, Baxter LT, Jain RK (1990) Interstitial pressure gradients in tissue-isolated and subcutaneous tumors: implications for therapy. Cancer Res 50: 4478-4484

Buchdunger E, Zimmermann J, Mett H, Meyer T, Muller M, Regenass U, Lydon NB (1995) Selective inhibition of the platelet-derived growth factor signal transduction pathway by a protein-tyrosine kinase inhibitor of the 2-phenylaminopyrimidine class. Proc Natl Acad Sci USA 92: $2558-2562$

Cummings J (1985) Method for determination of $4^{\prime}$-deoxydoxorubicin, $4^{\prime}$-deoxydoxorubicinol and their 7-deoxyaglycones in human serum by high-performance liquid chromatography. J Chromatogr 341: 401-409 
Furuhashi M, Sjoblom T, Abramsson A, Ellingsen J, Micke P, Li H, Bergsten-Folestad E, Eriksson U, Heuchel R, Betsholtz C, Heldin $\mathrm{CH}$, Ostman A (2004) Platelet-derived growth factor production by B16 melanoma cells leads to increased pericyte abundance in tumors and an associated increase in tumor growth rate. Cancer Res 64: 2725-2733

George D (2003) Targeting PDGF receptors in cancer - rationales and proof of concept clinical trials. Adv Exp Med Biol 532: 141 - 151

George DJ, Kaelin Jr WG (2003) The von Hippel-Lindau protein, vascular endothelial growth factor, and kidney cancer. $N$ Engl J Med 349: $419-421$

Griffon-Etienne G, Boucher Y, Brekken C, Suit HD, Jain RK (1999) Taxaneinduced apoptosis decompresses blood vessels and lowers interstitial fluid pressure in solid tumors: clinical implications. Cancer Res 59: $3776-3782$

Heldin CH, Rubin K, Pietras K, Ostman A (2004) High interstitial fluid pressure - an obstacle in cancer therapy. Nat Rev Cancer 4: 806-813

Heuchel R, Berg A, Tallquist M, Ahlen K, Reed RK, Rubin K, Claesson-Welsh L, Heldin CH, Soriano P (1999) Platelet-derived growth factor beta receptor regulates interstitial fluid homeostasis through phosphatidylinositol-3' kinase signaling. Proc Natl Acad Sci USA 96: $11410-11415$

Jain RK (1996) 1995 Whitaker Lecture: delivery of molecules, particles, and cells to solid tumors. Ann Biomed Eng 24: 457-473

Jain RK (2001) Delivery of molecular and cellular medicine to solid tumors. Adv Drug Deliv Rev 46: 149-168

Jain RK (2005) Normalization of tumor vasculature: an emerging concept in antiangiogenic therapy. Science 307: $58-62$

Jain RK, Baxter LT (1988) Mechanisms of heterogeneous distribution of monoclonal antibodies and other macromolecules in tumors: significance of elevated interstitial pressure. Cancer Res 48: 7022-7032

Kawai T, Hiroi S, Torikata C (1997) Expression in lung carcinomas of platelet-derived growth factor and its receptors. Lab Invest 77: 431 - 436

Kim SJ, Uehara H, Yazici S, Langley RR, He J, Tsan R, Fan D, Killion JJ, Fidler IJ (2004) Simultaneous blockade of platelet-derived growth factorreceptor and epidermal growth factor-receptor signaling and systemic administration of paclitaxel as therapy for human prostate cancer metastasis in bone of nude mice. Cancer Res 64: 4201-4208

Kong G, Anyarambhatla G, Petros WP, Braun RD, Colvin OM, Needham D, Dewhirst MW (2000) Efficacy of liposomes and hyperthermia in a human tumor xenograft model: importance of triggered drug release. Cancer Res 60: $6950-6957$

Matei D, Chang DD, Jeng MH (2004) Imatinib mesylate (Gleevec) inhibits ovarian cancer cell growth through a mechanism dependent on plateletderived growth factor receptor alpha and Akt inactivation. Clin Cancer Res 10: $681-690$

Netti PA, Hamberg LM, Babich JW, Kierstead D, Graham W, Hunter GJ, Wolf GL, Fischman A, Boucher Y, Jain RK (1999) Enhancement of fluid filtration across tumor vessels: implication for delivery of macromolecules. Proc Natl Acad Sci USA 96: 3137-3142
Ostman A (2004) PDGF receptors-mediators of autocrine tumor growth and regulators of tumor vasculature and stroma. Cytokine Growth Factor Rev 15: $275-286$

Pietras K, Ostman A, Sjoquist M, Buchdunger E, Reed RK, Heldin $\mathrm{CH}$ Rubin K (2001) Inhibition of platelet-derived growth factor receptors reduces interstitial hypertension and increases transcapillary transport in tumors. Cancer Res 61: 2929-2934

Ponce AM, Viglianti BL, Yu D, Yarmolenko PS, Michelich CR, Woo J, Bally MB, Dewhirst MW (2007) Magnetic resonance imaging of temperaturesensitive liposome release: drug dose painting and antitumor effects. J Natl Cancer Inst 99: 53-63

Schiller JH, Harrington D, Belani CP, Langer C, Sandler A, Krook J, Zhu J, Johnson DH (2002) Comparison of four chemotherapy regimens for advanced non-small-cell lung cancer. $N$ Engl J Med 346: 92 - 98

Tong RT, Boucher Y, Kozin SV, Winkler F, Hicklin DJ, Jain RK (2004) Vascular normalization by vascular endothelial growth factor receptor 2 blockade induces a pressure gradient across the vasculature and improves drug penetration in tumors. Cancer Res 64: 3731-3736

Truskey GA, Yuan F, Katz DF (2004) Transport phenomena in biological systems. Pearson Prentice Hall: Upper Saddle River, NJ

Viglianti BL, Abraham SA, Michelich CR, Yarmolenko PS, MacFall JR, Bally MB, Dewhirst MW (2004) In vivo monitoring of tissue pharmacokinetics of liposome/drug using MRI: illustration of targeted delivery. Magn Reson Med 51: 1153-1162

Vlahovic G, Rabbani ZN, Herndon II JE, Dewhirst MW, Vujaskovic Z (2006) Treatment with Imatinib in NSCLC is associated with decrease of phosphorylated PDGFR-beta and VEGF expression, decrease in interstitial fluid pressure and improvement of oxygenation. $\mathrm{Br} J$ Cancer 95: $1013-1019$

Willett CG, Boucher Y, di Tomaso E, Duda DG, Munn LL, Tong RT, Chung DC, Sahani DV, Kalva SP, Kozin SV, Mino M, Cohen KS, Scadden DT, Hartford AC, Fischman AJ, Clark JW, Ryan DP, Zhu AX, Blaszkowsky LS, Chen HX, Shellito PC, Lauwers GY, Jain RK (2004) Direct evidence that the VEGF-specific antibody bevacizumab has antivascular effects in human rectal cancer. Nat Med 10: 145-147

Winkler F, Kozin SV, Tong RT, Chae SS, Booth MF, Garkavtsev I, Xu L, Hicklin DJ, Fukumura D, di Tomaso E, Munn LL, Jain RK (2004) Kinetics of vascular normalization by VEGFR2 blockade governs brain tumor response to radiation: role of oxygenation, angiopoietin-1, and matrix metalloproteinases. Cancer Cell 6: 553-563

Yuan F (1998) Transvascular drug delivery in solid tumors. Semin Radiat Oncol 8: $164-175$

Yuan F, Dellian M, Fukumura D, Leunig M, Berk DA, Torchilin VP, Jain RK (1995) Vascular permeability in a human tumor xenograft: molecular size dependence and cutoff size. Cancer Res 55: 3752-3756

Yuan F, Leunig M, Huang SK, Berk DA, Papahadjopoulos D, Jain RK (1994) Microvascular permeability and interstitial penetration of sterically stabilized (stealth) liposomes in a human tumor xenograft. Cancer Res 54: $3352-3356$ 